\title{
Questes
}

vestes Revue pluridisciplinaire d'études médiévales

\section{Rituels du quotidien dans les dits amoureux}

\section{Laëtitia Tabard}

\section{(2) OpenEdition \\ Journals}

Édition électronique

URL : http://journals.openedition.org/questes/3421

DOI : 10.4000/questes.3421

ISSN : 2109-9472

Éditeur

Les Amis de Questes

Édition imprimée

Date de publication : 15 septembre 2008

Pagination : 52-63

ISSN : 2102-7188

\section{Référence électronique}

Laëtitia Tabard, "Rituels du quotidien dans les dits amoureux », Questes [En ligne], 15 | 2008, mis en ligne le 01 janvier 2014, consulté le 01 mai 2019. URL : http://journals.openedition.org/questes/3421 ; DOI : 10.4000/questes.3421 


\title{
Rituels du quotidien dans les dits amoureux
}

\author{
Laëtitia TABARD
}

Si le quotidien semble avoir toute sa place dans la littérature satirique ou comique, souvent comme envers de l'idéalisation, suscitant le rire par le décalage entre le sujet et sa stylisation, il est plus délicat de juger de la valeur que lui confère la littérature courtoise. La représentation des activités quotidiennes peut-elle jouer autrement que comme contraste? Écrire le quotidien permet-il de conférer une dignité littéraire à ce qui apparait d'abord comme une matière déplacée dans le contexte amoureux?

Le Livre du Voir Dit de Guillaume de Machaut, et le Dit de Poissy de Christine de Pizan, deux textes de la littérature courtoise, se signalent par leur ambivalence stylistique et ont fait l'objet de nombreuses remarques critiques concernant leur «réalisme», notamment en raison de la place qu'y occupent les activités quotidiennes. Dans le premier ouvrage, le narrateur presse la jeune fille qu'il aime de s'acquitter d'un vœu et d'accomplir un pèlerinage afin de pouvoir passer du temps avec elle : ils se rendent alors à Saint-Denis, le jour de la fête du Lendit, passent une joyeuse journée à La Chapelle, et demeurent quelques jours ensemble. Dans Le Dit de Poissy, Christine de Pizan fait le récit d'une visite à l'abbaye de Poissy: la narratrice convie une troupe d'amis à venir avec elle rendre visite à sa fille, qui est religieuse. Après avoir exploré les lieux, la joyeuse compagnie passe une soirée agréable à l'hôtel de Bourbon. Un débat surgit lors du retour entre une dame et un chevalier qui prétendent tous deux souffrir au plus haut point, à cause d'un amour perdu. Dans les deux cas, le discours amoureux se trouve pris dans le temps 
d'un voyage dans un lieu réel, qui ouvre à la narration et à l'interprétation l'espace de la vie quotidienne d'une communauté.

\section{L'inscription du quotidien dans le dit: le cadre du récit}

Le genre complexe du dit, aussi divers dans ses thèmes que dans les formes d'écriture qu'il déploie, se rattache à une expérience du temps qui permet la référence au quotidien : discours à la première personne, prise de parole, il se trouve situé dans le temps même de son énonciation, "par rapport à l'ici et au maintenant ${ }^{1}$. Cet ancrage dans la réalité spatiale et temporelle est clairement marqué dans Le Livre du Voir Dit, où le pèlerinage se déroule un jour précis :

Ce fu droit le jour que l'en dit

La beneyçon du Lendit'.

La rime rappelle l'étymologie de Lendit, qui vient de «indictus », et souligne l'importance de cette indication temporelle qui assigne à l'événement un jour fixe ${ }^{3}$. Le voyage est plus précisément daté dans Le Dit de Poissy, et Christine de Pizan insiste sur cette précision lorsqu'elle présente son œuvre au grand seigneur qui en est le dédicataire :

Comme il avint vous orrés sans targer

Et en quel temps,

La ou ce fu vous sera dit par temps,

Car il n'a pas ne mille ne cent ans,

Non pas un mois ; ains fu en l'esbatant

Gracieux mois

${ }^{1}$ Jacqueline Cerquiglini-TOUlet, «Le Clerc et l'écriture: le Voir Dit de Guillaume de Machaut», Grundriss der romanischen Literaturen des Mittelalters, Begleitreihe 1, Heidelberg, Winter, 1980, p. 165.

${ }^{2}$ Guillaume de machaut, Le Livre du Voir Dit, éd. Paul Imbs, revue par Jacqueline Cerquiglini-Toulet, Paris, L.G.F., «Lettres gothiques », 1999, v. 3589-3590, p. 332.

${ }^{3}$ La date ne figure pas précisément dans le texte, mais peut se déduire de la mention de certains faits : l'action du Livre du Voir Dit semble se dérouler de 1362 à 1365, selon Paul Imbs (voir Paul ImBS, Le Voir Dit de Guillaume de Machaut, étude littéraire, Paris, Klincksieck, 1991, p. 33-54). 
D'avril le gay, ou reverdissent bois,

Ce present an mil .cccc. ainçois

La fin du mois ${ }^{4}$.

Le jour de la visite est situé dans le temps historique, mais aussi par rapport au moment où le livre est écrit ; l'auteur établit un lien étroit avec le présent.

Cet ancrage temporel permet une «authentification» de l'événement, qui correspond à l'ambition de vérité affichée par le titre même du Livre du Voir Dit. On peut aussi interpréter cette référence comme volonté de faciliter l'impression dans la mémoire ${ }^{5}$, comme semble l'indiquer Machaut :

Et pour ce qu'il en fust memoire

Ainsi allames par la foire...

En se référant à la célèbre foire du Lendit, qui s'ouvre par la bénédiction de l'archevêque de Paris, le onze juin, Guillaume de Machaut inscrit un élément de son histoire individuelle dans la mémoire collective, et les actions les plus banales se mêlent à l'événement mémorable.

Le cadre de la ville donne cependant au récit les couleurs de la vie quotidienne. Des figures de milieux divers apparaissent dans les deux récits : les personnages du Livre du Voir Dit pénètrent dans l'espace de la ville, côtoient les marchands de la foire, rencontrent lors du repas à La Chapelle un sergent d'armes ivre qui les conduit dans la demeure d'un «villain » ${ }^{7}$, et soupent en compagnie de musiciens. Les personnages semblent entourés d'une foule anonyme et bigarrée. La référence à d'autres milieux sociaux est moins claire chez Christine de Pizan, qui insiste plutôt sur la noblesse des membres de la famille royale rencontrés dans le «bel hostel royal» de l'abbaye de Poissy, Marie de Bourbon et «la tres noble

\footnotetext{
${ }^{4}$ Christine de Pizan, Le Livre du Dit de Poissy, éd. Barbara Altmann, The Love Debate Poems of Christine de Pizan, Gainesville, University Press of Florida, 1998, v. 31-39, p. 206, 207.

${ }^{5}$ Le rôle du quotidien, et notamment de l'objet, dans la mémorisation est souligné par MarieThérèse LORCIN et Danièle AlEXANDrE-BIDOn, dans Le Quotidien au temps des fabliaux. Textes, images, objets, Paris, Picard, 2003, p. 97.

${ }^{6}$ Le Livre du Voir Dit, éd. cit., v. 3601-3602, p. 334.

${ }^{7}$ Ibid., v. 3640, p. 336.
} 
princesse, / Fille du roy $»^{8}$, Marie. La narratrice cependant se présente également en « compagnie plaisant $»^{9}$, et à l'hôtel où " gent s'amasse »" la troupe de ses amis se livre aux plaisirs de la danse et des chants en compagnie de «jeune gent et de joyeuse entente », « de noz gens » et « d'autre gent, trestous mignos et gens » ${ }^{11}$. Ce mélange social est celui de la ville, «tiers monde qui n'est ni celui du clerc ni celui du chevalier ${ }^{12}$. Les récits se déroulent tous les deux dans des lieux urbains clairement identifiés, dans le titre même du Dit de Poissy, et dans le récit du Voir Dit qui mentionne « La Chapelle ». Le récit mêle alors intimement l'histoire personnelle et la vie collective, les personnages participent aux activités du groupe social.

Le quotidien, dans ce montage narratif caractéristique du dit, prend la forme d'une séquence narrative particulière. Les critiques ${ }^{13}$ ont relevé à de nombreuses reprises la division formelle du Dit de Poissy, qui oppose deux parties bien distinctes, l'une consacrée à la visite de l'abbaye, l'autre au débat amoureux qui surgit entre un écuyer et une dame, sur le chemin du retour. Paul Imbs relève également dans Le Livre du Voir Dit l'importance du mélange dans le récit du séjour à Saint-Denis et à La Chapelle, qui «marque le sommet de l'action romanesque et de l'art de Guillaume de Machaut conteur, mélangeant habilement, et en apparence pour le seul plaisir, la succession rapide des faits dominants de la rencontre ou adjacents [...], les commentaires psychologiques mais aussi moraux d'une inhabituelle

${ }^{8}$ Le Dit de Poissy, éd. cit., v. 274-275, p. 212.

${ }^{9}$ Ibid., v. 54, p. 207.

${ }^{10}$ Ibid., v. 603, p. 221.

${ }^{11}$ Ibid., v. 756-761, p. 224-225.

${ }^{12}$ Jacqueline CerQuiglini-TOUlet, Un Engin si soutil: Guillaume de Machaut et l'écriture, Paris Champion, 2001 (1 $1^{\text {ère }}$ éd. 1985), p. 130.

${ }^{13}$ Voir les deux articles de Liliane Dulac, «Le Livre du dit de Poissy de Christine de Pizan. Poème éclaté ou montage signifiant », dans Écrire pour dire, études sur le dit médiéval, éd. Bernard RIBEMONT, Paris, Klincksieck, 1990, et "Poème mondain ou oeuvre morale ? L'ambiguïté d'un dit de Christine de Pizan ", dans La Protection spirituelle au Moyen Agge, Cabiers de recherches médiévales, vol. VIII, Orléans, CEMO (Centre d'études médiévales d'Orléans), 2001, ainsi que l'article de Barbara Altmann, «Diversity and Coherence in Christine de Pizan's Dit de Poissy », French Forum, septembre 1987, p. 261-271. 
sobriété, et un minimum d'accompagnement lyrique (une ballade) pour traduire l'état d'âme du poète exalté, que confirmera peu après le rondeau de la dame ${ }^{14}$. »

La présence de la vie quotidienne donne clairement l'impression que des éléments hétérogènes, «adjacents ", pénètrent le dit amoureux. On retrouve l'effet de décalage thématique, mais le temps du quotidien n'est plus seulement introduit comme un contrepoint : il forme le cadre même du récit.

\section{$\underline{L}$ a représentation du temps social : le quotidien comme rituel}

Les étapes du récit, dans les deux textes, sont marquées par la mention des repas, qui scandent le temps de la vie quotidienne. Ce rythme propre à la vie sociale se substitue même en partie au temps religieux dans Le Livre du Voir Dit: l'accomplissement du pèlerinage apparaît comme une simple étape du déplacement des personnages, et le vœu acquitté bien vite, «sans targier», fait l'objet d'une courte phrase :

Si le paiames sans targier.

Et puis nous venimes mangier

A une ville qu'on appelle

Par tout a Paris la Chapelle ${ }^{15}$.

Pris dans la succession temporelle que soulignent les adverbes «si» et «puis», le pèlerinage se trouve rapidement relégué au second plan, alors que la narration s'attarde sur le repas. Celui-ci est suivi d'un temps de repos, la dame éprouvant le besoin de dormir quelque temps; après quoi les personnages retrouvent de joyeux compagnons, se divertissent en allant « jouer aus boules $»^{16}$, et soupent joyeusement dans un verger, en se livrant au plaisir de la musique et des chants jusque tard dans la nuit. La journée est donc organisée en fonction des

\footnotetext{
${ }^{14}$ Paul IMBS, op. cit., p. 42.

${ }^{15}$ Le Livre du Voir Dit, éd. cit., v. 3613-3616, p. 334.

${ }^{16}$ Ibid., v. 3777 , p. 344
} 
rythmes du corps, selon la partition entre nourriture et dépense physique. Les heures canoniales sont à peine mentionnées, si ce n'est comme point de repère ponctue ${ }^{17}$. Le sentiment du sacré se transpose dans le domaine amoureux et le repas auprès de la dame acquiert en retour une connotation religieuse :

Et delés ma dame disnai

Ou petit prins pain et vin ai,

Qu'en li veoir me delitoie

Et de ce la me saouloie ${ }^{18}$...

La métaphore de la nourriture pour les «biens d'amour ${ }^{19}$ transforme le repas en une nourriture spirituelle et rappelle l'eucharistie.

Le quotidien apparaît sous la forme de la répétition, ce qui le rapproche aussi du rituel religieux. La reprise de certains termes souligne la continuité temporelle tout en marquant clairement le changement par des effets de variation : le vœu de pèlerinage est acquitté «pa[yé] » ${ }^{20}$, de même que l'hôte :

Apres mengier l'oste paiames

Et puis d'illuecques nous levames ${ }^{21}$.

Cette dernière phrase est reprise presque textuellement lorsque les personnages s'éveillent après s'être reposés :

Quant temps fu, d'ilec nous levasmes

Et pluiseurs compagnons trouvames

Qui en chantant nous esveillierent ${ }^{22} \ldots$

${ }^{17}$ Encore celui-ci n'est-il que proposé par le poète qui prétend ne pas dormir avec sa dame :

Et la je vous attenderai

Et vous esveillerai a nonne

Si tost com j'orrai qu'on la sonne. (v. 3668-3670, p. 338)

Finalement, le chant des «compagnons », de ceux qui partagent le pain du repas, se substitue à la sonnerie de l'heure religieuse.

${ }^{18}$ Ibid., v. 3825-3828, p. 346.

${ }^{19}$ La métaphore de la nourriture, qui évoque la satisfaction de l'amant, parcourt toute la séquence. Ibid., p. 342.

${ }^{20}$ Ibid., v. 3613, p. 334.

${ }^{21}$ Ibid., v. 3625-3626, p. 334. 
Cet éveil trouve un écho dans la veille prolongée de toute la compagnie :

La jusques prés du jour veillames ${ }^{23}$.

Le sentiment du quotidien se révèle inséparable du sentiment de la répétition et de la durée continue. Or les temps forts de ces journée rappellent ceux du rituel religieux où l'on communie, se lève, chante, veille.

Dans Le Dit de Poissy, où la visite à l'abbaye tient une place bien plus grande que le pèlerinage chez Machaut, le temps ne se mesure pas non plus en fonction des heures liturgiques mais à partir des repas pris en commun; pourtant ceux-ci sont associés à des rituels qui montrent la prégnance du religieux. À l'arrivée au couvent, les personnages vont entendre la messe ${ }^{24}$, puis sont conviés à se restaurer, à boire et à «desjuner $»^{25}$. Le fait de se laver les mains, après les repas, apparaît comme un rite de purification lié à la pratique religieuse dans les vers qui évoquent le dîner ${ }^{26}$ et surtout le souper :

... Et adonc du mengier

Sommes levé,

Dites graces aprés qu'osmes lavééc.

La journée, enfin, s'achève sur une promenade ${ }^{28}$. Cette fin reprend dans un ordre inverse les actions du matin, où se succédaient le voyage, la messe, et le déjeuner. Elle souligne la circularité de ce temps social fortement ritualisé.

Les activités quotidiennes dessinent donc plus que les références au temps le cadre à la fois temporel et spatial du récit, parce qu'elles constituent un rituel, qu'on peut définir comme l'association d'une action, d'un lieu et d'un moment, qui se

\footnotetext{
${ }^{22}$ Ibid., v. 3773-3775, p. 344.

${ }^{23}$ Ibid., v. 3811, p. 346.

${ }^{24}$ Le Dit de Poissy, éd. cit., v. 239-240, p. 212

${ }^{25}$ Ibid., v. 241, p. 212, v. 348-353, p. 214.

${ }^{26}$ Ibid., v. 620-625, p. 221.

${ }^{27}$ Ibid., v. 867-869, p. 227.

${ }^{28}$ Ibid., v. 870-884, p. 227-228.
} 
répète selon un ordre codifié, régulier; cette représentation du temps marque la valeur des gestes du quotidien dans la continuité de l'ordre et des pratiques du monde religieux.

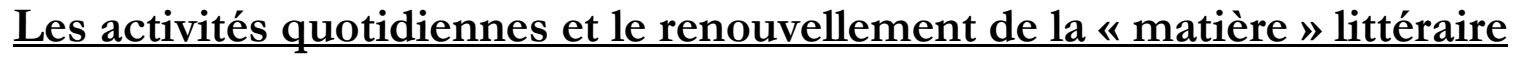

On hésite sur le sens de cette représentation qui semble sortir du cadre symbolique ou des motifs traditionnels. Cet effet est particulièrement clair lorsque la narratrice du Dit de Poissy décrit l'espace domestique: ainsi les matelas sur lesquels les nonnes couchent évoquent leurs habitudes, mais la description oscille entre l'éloge, qui recourt à des motifs connus, et une vision plus précise :

Si n'y vestent chemises, et sus langes

Gisent de nuit ; n'ont pas coustes a franges

Mais materas

Qui sont couvers de beaulx tapis d'Arras

Bien ordenez, mais ce n'est que baras,

Car ilz sont durs et emplis de bourras ;

Et la, vestues,

Gisent de nuis celles dames rendues,

Qui se lievent ou elles sont batues

A matines. La leurs chambres tendues

En dortouer

Ont pres a pres, et en refretouer

Disnent tout temps, ou a beau lavouer ${ }^{29}$.

L'effet de répétition, mise en valeur par le rejet de l'expression «gisent de nuit» mais aussi par la locution «tout temps», évoque le rythme du temps liturgique qui fait le quotidien de la religieuse. Mais ce qui frappe avant tout, c'est le détail précis sur la matière, le «bourras », qui est juxtaposé à la mention des « beaux tapis », et crée une opposition nette entre apparence et réalité. Chez Machaut, on

${ }^{29}$ Ibid., v. 314-326, p. 213-214. 
trouve un effet analogue dans des allusions de détail, par exemple pour un sergent qui conduit le poète et les dames dans la chambre où ils pourront se reposer :

Il avoit la un sergent d'armes,

Qui avoiet beu jusques aus larmes

D'un trop bon vin de Saint Poursain :

Chascun le tesmoingne pour sain,

Mais il le faisoit chanceller

Si qu'il ne s'en pooit celer ${ }^{30}$.

La mention de l'origine du vin introduit une référence concrète, mais il s'agit aussi d'un élément topique. Les noms propres, les références aux localités d'où proviennent les aliments, comme le vin de «Saint Poursain» ou les «frommages de Brye $»^{31}$, inscrivent dans le texte le monde du quotidien, mais ces références peuvent également jouer comme des citations d'autres textes et ne sont pas forcément destinées à créer un effet de réel ; on retrouve dans le Dit de Poissy des termes qui sonnent comme des rappels, par exemple le don, de la part de la prieure, d'un bon vin de «Saint Pourçain», apporté par un «sergent» ${ }^{32}$. Machaut, cependant, déjoue l'association trop attendue entre « saint Poursain » et «sain » par un détail révélateur sur la démarche du sergent, et joint à la représentation traditionnelle la vérité d'une observation concrète, qui dévoile ce que l'on veut « celer ».

Cette ambivalence est aussi liée à un double point de vue sur les activités du quotidien, qui relèvent à la fois du social et de l'intime. Dans le Dit de Poissy, le rythme des activités du jour incarne la discipline à laquelle les nonnes se soumettent; il est un rappel à l'ordre, qui interrompt le sommeil, ou le plaisir d'être ensemble. Le souper lui-même marque la fin des réjouissances :

Chacun chanta tant qu'il fu pres de nuis,

${ }^{30}$ Le Livre du Voir Dit, éd. cit., v. 3633-3638, p. 336.

${ }^{31}$ Ibid., v. 3778-3781, p. 344.

${ }^{32}$ Voir les vers 776 et 783, p. 225 du Dit de Poissy, éd. cit. 
Car le dancier ne tournoit a annuis

A nul qui fust. Si fu le souper cuis,

Ce nous dist on,

Adonc de la dance nous departons $[\ldots]^{33}$

Une opposition structurante se dessine alors dans le temps de la journée ellemême entre un temps ritualisé, fait d'obligations, et des moments libres ; mais la vie individuelle demeure en arrière-plan des actions collectives et se dévoile par quelques vers suggestifs. Ainsi, à l'heure du dîner, l'image de la fille et de la mère se tenant par la main apparaît :

Et ma fille, qui toudis par la main

M'aloit tenant, de cuer de desir plain

Moult me prioit a jusque a lendemain

De sejourner,

Et retourner leans apres disner

Nous voulsissions ; adont falu finer

Notre parler et notre erre ordener ${ }^{34}$.

La plainte personnelle est bien vite étouffée sous les impératifs de la vie collective $^{35}$, mais la représentation des activités quotidiennes mêle ces deux aspects de la vie courante. Le récit du Livre du Voir Dit fait ainsi alterner temps intimes et temps collectifs. Après le souper animé sur lequel se clôt la première journée, le poète se réjouit de pouvoir, pendant sept jours, dîner auprès de sa dame «priveement $»^{36}$; on retrouve cette opposition du privé et du public au moment où le poète prend congé de son amie, qui souhaite le retrouver à son lever, "au matinet $[\ldots]$ / Et non pas cy devant la gent ${ }^{37}$.» Ce temps de la vie intime ne semble pas avoir de place précise dans la journée, mais il est lié à l'espace de la chambre, au moment du sommeil, l'après-midi, ou le matin.

${ }^{33}$ Ibid., v. 749-753, p. 224.

${ }^{34}$ Ibid., v. 585-591, p. 220.

35 De même, le débat au cours duquel s'exprime le sentiment personnel s'interrompt soudainement au moment de l'arrivée à Paris : voir ibid., v. 2053, p. 257.

${ }^{36}$ Le Livre du Voir Dit, éd. cit., v. 3829, p. 346

${ }^{37}$ Ibid., v. 3893-3894, p. 350. 
On peut dès lors s'interroger sur la valeur de la référence au rythme répétitif de la vie quotidienne dans le dit amoureux, car en modelant le récit sur le temps social, tout en faisant sentir le temps subjectif, les deux poètes font aussi de la vie quotidienne l'apparence qui dissimule et révèle la vie intime sous-jacente. Certains indices suggèrent que le quotidien dissimule d'autres intrigues. Chacun des protagonistes entreprend en effet de voyager en ayant à l'esprit un but strictement privé, cherchant le plaisir de la compagnie d'une personne aimée, dimension qui introduit une tout autre perspective : le poète rêve de se trouver seul avec sa dame $^{38}$, et Christine de Pizan désire voir sa fille ${ }^{39}$. Le récit suit donc les fils entrelacés de deux intrigues. Avec un certain humour, ces arrière-pensées, décelables derrière l'apparente gratuité des faits, sont indiquées dès le départ par Guillaume de Machaut :

Et pour ce qu'il en fust mémoire, Ainsi alames par la foire,

Ou moult de choses marchandames,

Mais onques rien n'i achetames,

Car certainement nos pensees

Estoient ailleurs ordennees :

C'estoit a son pellerinage... ${ }^{40}$

Cette conjugaison de motifs suggère une trame sous-jacente. Certains réseaux lexicaux se chargent de valeurs multiples en jouant sur les différents sens d'un même mot et sur ses connotations. Le paiement de la dette, qui appartient au domaine économique et évoque le règlement du repas, devient une métaphore pour le pèlerinage acquitté par la dame, comme pour l'amour, qui ce jour-là «paia » le poète « le bon jour qu'[il lui] devoit ».

\footnotetext{
${ }^{38}$ Ibid., v. 3555-3560, p. 330.

${ }^{39}$ Le Dit de Poissy, éd. cit., v. 39-50, p. 207.

${ }^{40}$ Le Livre du Voir Dit, éd. cit., v. 3601-3607.
} 
Les activités du quotidien occupent dès lors une place qui n'est plus seulement annexe dans le dit amoureux. Le lien de l'œuvre littéraire et du concret assure sa résurgence dans la mémoire, accrédite le propos amoureux qui devient un Voir Dit. Si le sentiment d'un contraste demeure entre le monde des amants et celui des activités courantes, ce décalage ne semble pas source de parodie ou d'effets comiques : il met au premier plan, par un récit qui s'appuie sur le rythme même des activités collectives, le sentiment d'un temps ritualisé et codifié, et fait d'autant mieux sentir le jeu sous-jacent des désirs individuels et du temps intime, privé. Cette double représentation du quotidien confère une grande richesse aux détails dits « réalistes » par lesquels les actions quotidiennes s'écrivent. Dans le jeu de la métaphore, des références intertextuelles, des connotations, le simple fait de se promener, de manger et de boire, ou de dormir, se charge de sens symboliques et s'agrège à la matière courtoise. 